\title{
Monthly VLBI Monitoring of Selected Gamma-Bright Quasars
}

\author{
S. Britzen ${ }^{1}$, A. Witzel, T. P. Krichbaum \\ Max-Planck-Institut für Radioastronomie, Bonn, Germany.
}

J. Roland

CNRS, Inst. d'Astrophysique, Paris, France; Leiden observatory, Netherlands.

S. J. Wagner

Landessternwarte Heidelberg, Königstuhl, 69117 Heidelberg, Germany.

Abstract. In the past few years EGRET observations of extragalactic radio sources demonstrated the presence of blazars at high energies and supplied informations about their enormous luminosities. Questions arose whether outbursts at these high frequencies can be linked to structural changes in the radio regime and the need for VLBI monitoring became obvious. In the past two decades geodetic VLBI measurements gathered this kind of data.

We analyzed $\lambda=3.6 \mathrm{~cm}$ observations with high time-resolution (up to once a month) from the geodetic IRIS campaigns for the $\gamma$-ray active quasars PKS 0420-014 and PKS 0528+134. In both sources superluminally moving jet components could be traced over time periods of several years on curved trajectories. Both sources exhibit component ejections preceding the time of an observed gamma-ray flaring.

\section{PKS 0420-014}

PKS 0420-014 was the first source to exhibit a simultaneous gamma-optical flaring: over five years time the source showed quasi-periodic optical flaring; the last flaring (Feb./March 1992) coincided with an outburst observed in the gamma-ray regime (Wagner et al. 1995). The "lighthouse" model (Camenzind \& Krockenberger 1992) has been applied to explain the observed variability features. It makes clear cut predictions concerning the VLBI structure of the source. According to this model superluminal motion should be observable for jet components all of them following one common helical path. To verify these prognoses we investigated the structural bearings of the source at nine $3.6 \mathrm{~cm}$ VLBI-observations (IRIS-S) between 1989.32 and 1992.48. PKS 0420-014 reveals a typical core-jet morphology at this wavelength showing a jet which extends about 5 mas to the south consisting of at least four jet components which move outwards with apparent superluminal speeds (see Fig. $2 ; 7.9 \mathrm{c}, 5.3 \mathrm{c}, 3.9 \mathrm{c}$ and $2.1 \mathrm{c} ; H_{0}=100$ $\left.\mathrm{km} \mathrm{s}^{-1} \mathrm{Mpc}^{-1}, q_{0}=0.5\right)$. The decline in velocity as a function of core distance is significant. The extrapolated time of ejection coincides for at least three of the components with minima preceding total flux-density outbursts in the radio and the origin of the newest components precedes the dates of $\gamma$-ray flares by several months. All individual component paths can be represented most easily by one common path. Thus, the predictions of the lighthouse model could be confirmed.

An alternative explanation of the variability and morphology seen in the VLBI data can be given in the frame of the two-fluid model by a precession of the accretion disk due to a binary system (Roland et al. 1994).

\footnotetext{
${ }^{1}$ Present address: NFRA, 7991 PD Dwingeloo, Netherlands.
} 


\section{PKS 0528+134}

PKS $0528+134$ is one of the brightest gamma-ray sources in the sky and the second most distant quasar detected by EGRET. The high-energy output of this source is huge and extreme flux- and spectral variability have been reported (Mukherjee et al. 1996). To investigate the structural behavior of PKS 0528+134 we selected 20 geodetic VLBI-observations (IRIS-S) spaced between 1986.25 and 1994.07. By fitting gaussian components to the data, we received parameter sets describing the structural appearance at the individual epochs. We detected rapid structural variability in the jet of PKS $0528+134$. At least four jet components clearly reveal apparent superluminal motion (see Fig. 1; component b to e: $5.5 \pm 1.4 ; 4.7 \pm 0.4 ; 5.2 \pm 0.4 ; 5.2 \pm 0.6)$. The two most recently ejected components may be related to outbursts in the gamma-ray regime. Different component pathes for adjacent components have been observed, the pathes revealing strong curvature in the vicinity of the core. mm-VLBI observations (see Krichbaum et al., these Proceedings, p. 37) yield complementary results.

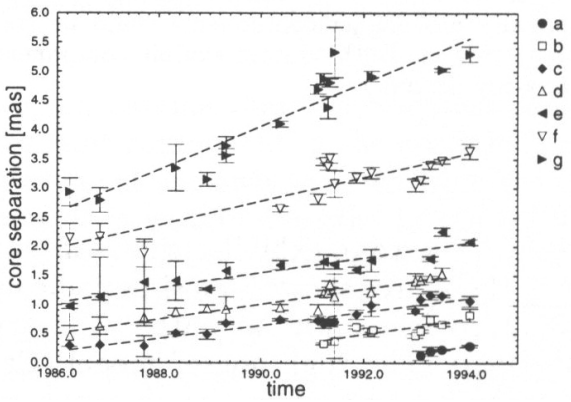

Figure 1. Component separations as a function of time for PKS $0528+134$.

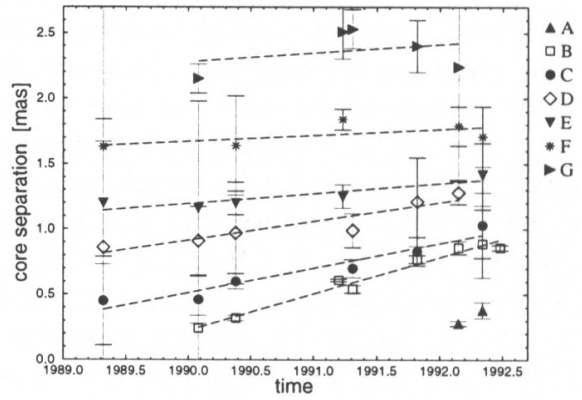

Figure 2. Component separations as a function of time for PKS 0420-014.

\section{References}

Camenzind, M., \& Krockenberger, M. 1992. A 6 A, 255, 59-62.

Mukherjee, R., et al. 1996. ApJ, 470, 831-838.

Roland, J., Teyssier, R., \& Roos, N. 1994. A\&A, 290, 357-363.

Wagner, S. J., et al. 1995. $A \mathscr{E} A, \mathbf{2 9 8}, 688-698$. 